\title{
Consolidative allogeneic hematopoietic stem cell transplantation after chimeric antigen receptor T-cell therapy for relapsed/refractory B-cell acute lymphoblastic leukemia: who? When? Why?
}

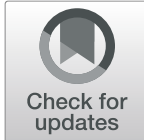

Huiwen Jiang ${ }^{1,2}, \mathrm{Yu} \mathrm{Hu}^{1,2}$ and Heng $\mathrm{Mei}^{1,2^{*}}$ (i)

\begin{abstract}
Although anti-CD19 chimeric antigen receptor (CAR) T-cell therapy shows good efficacy in patients with relapsed/ refractory B-cell acute lymphoblastic leukemia ( $r / r$ B-ALL), it fails to improve long-term leukemia-free survival (LFS). Allogeneic hematopoietic stem cell transplantation (allo-HSCT) after CAR T-cell therapy has emerged as a promising strategy to prolong LFS. Nevertheless, which patients are likely to benefit from consolidative allo-HSCT, as well as the optimal therapeutic window, remain to be explored. Recent clinical data indicate that patients with complex karyotypes, adverse genes, and high pre-infusion minimal residual disease (MRD) by flow cytometry in the bone marrow, were at high risk of relapse after CAR T-cell therapy. High pre-lymphodepletion lactate dehydrogenase, low prelymphodepletion platelet count, absence of fludarabine in lymphodepletion, persistent leukemic sequence by high throughput sequencing in bone marrow after CAR T-cell infusion, and early loss of CAR T cells have also been linked to relapse after CAR T-cell therapy. In patients having these risk factors, consolidative allo-HSCT after CAR T-cell therapy may prolong LFS. Allo-HSCT provides optimal clinical benefit in patients with MRD-negative complete remission, typically within three months after CAR T-cell therapy. Herein, we summarize the clinical data on consolidative allo-HSCT after anti-CD19 CAR T-cell therapy, as well as the potential factors associated with allo-HSCT benefit. We also discuss the optimal therapeutic window and regimen of consolidative allo-HSCT. Finally, and most importantly, we provide recommendations for the assessment and management of r/r B-ALL patients undergoing anti-CD19 CAR T-cell therapy.
\end{abstract}

Keywords: Chimeric antigen receptor-modified T-cell therapy, Allogeneic hematopoietic stem cell transplantation, Relapsed or refractory B-cell acute lymphoblastic leukemia, Prognosis

\footnotetext{
* Correspondence: hmei@hust.edu.cn

'Institute of Hematology, Union Hospital, Tongji Medical College, Huazhong University of Science and Technology, Wuhan 430022, China

${ }^{2}$ Hubei Clinical Medical Center of Cell Therapy for Neoplastic Disease, Wuhan 430022, China
}

C C The Author(s). 2020 Open Access This article is licensed under a Creative Commons Attribution 4.0 International License, which permits use, sharing, adaptation, distribution and reproduction in any medium or format, as long as you give appropriate credit to the original author(s) and the source, provide a link to the Creative Commons licence, and indicate if changes were made. The images or other third party material in this article are included in the article's Creative Commons licence, unless indicated otherwise in a credit line to the material. If material is not included in the article's Creative Commons licence and your intended use is not permitted by statutory regulation or exceeds the permitted use, you will need to obtain permission directly from the copyright holder. To view a copy of this licence, visit http://creativecommons.org/licenses/by/4.0/ The Creative Commons Public Domain Dedication waiver (http://creativecommons.org/publicdomain/zero/1.0/) applies to the data made available in this article, unless otherwise stated in a credit line to the data. 


\section{Background}

Patients with relapsed/refractory B-cell acute lymphoblastic leukemia ( $r / r$ B-ALL) often have clinicopathological characteristics associated with poor prognosis, such as high tumor burden and high-risk gene mutations. Conventional therapies typically fail to achieve minimal residual disease (MRD)negative complete remission (CR). However, advances in chimeric antigen receptor (CAR) T-cell therapy have dramatically improved the treatment of $r / r$ B-ALL [1-3] The first FDA-approved CD19-targeted CAR T-cell therapy, Tisagenlecleucel, has recently been established as the standard of care for $\mathrm{r} / \mathrm{r}$ B-ALL patients aged under 26 as per the NCCN Guidelines (https://www.nccn.org/) [4-6]. However, despite the unprecedented remission rate and controllable side effects, disease relapse after CAR T-cell therapy remains a significant challenge [7, 8]. Early loss or exhaustion of CAR T cells, selection of CD19-negative clones, downregulation of CD19 expression, lineage switch of leukemia, and tumor microenvironment are important factors contributing to relapse after CAR T-cell therapy $[9,10]$. Hence, the development of new therapeutic strategies that overcome these obstacles and achieve durable remission in $\mathrm{r} / \mathrm{r}$ B-ALL patients are urgently warranted.

For decades, allogeneic hematopoietic stem cell transplantation (allo-HSCT) has been regarded as the only wellestablished curative cellular therapy for patients with BALL. However, $r / r$ B-ALL patients with MRD cannot achieve a satisfactory response and have a higher relapse rate after allo-HSCT than patients without MRD [11-17]. Furthermore, $\mathrm{r} / \mathrm{r}$ B-ALL patients may develop severe organ damage or infection after aggressive chemotherapy and, hence, be precluded from allo-HSCT. For these r/r B-ALL patients with MRD, CAR T-cell therapy can serve as an effective and safe method to induce MRD-negative CR before subsequent allo-HSCT [18-25]. The combination of CAR T-cell therapy and allo-HSCT has been suggested to reduce the relapse rate of leukemia. Notably, several studies found that patients receiving consolidative allo-HSCT have longer leukemia-free survival (LFS) than the patients receiving CAR T-cell therapy alone [26-28]. Nonetheless, consolidative allo-HSCT is not recommended for all patients because it will increase the economic burden and bring risk of severe toxicity, such as graft-versus-host disease (GVHD). Besides, when and how to perform consolidative allo-HSCT are still not well defined. Therefore, clinicians should perform thorough benefit and risk assessment before using consolidative allo-HSCT in r/r B-ALL patients after CAR T-cell therapy.

Herein, we review the findings from large-scale clinical trials of CAR T-cell therapy and consolidative alloHSCT and summarize the potential factors associated with consolidative allo-HSCT benefit. Finally, we provide a recommendation to evaluate and transfer $\mathrm{r} / \mathrm{r}$ B-ALL patients to consolidative allo-HSCT.

\section{Anti-CD19 CAR T-cell therapy and consolidative Allo-HSCT for $r / r$ B-ALL}

In a number of clinical trials, some $\mathrm{r} / \mathrm{r}$ B-ALL patients received consolidative allo-HSCT after anti-CD19 CAR T-cell therapy. The patient characteristics, costimulatory domains of CAR $\mathrm{T}$ cells, lymphodepleting chemotherapies before CAR T-cell infusion, subsets and doses of infused CAR $\mathrm{T}$ cells, CR rates and duration after CAR T-cell therapy, and consolidative allo-HSCT application methods are summarized in Table 1.

KTE-C19 (axicabtagene ciloleucel) safety and efficacy were assessed in a phase 1 clinical trial of 20 pediatric and young adult $\mathrm{r} / \mathrm{r}$ B-ALL patients at the National Cancer Institute [21]. Infusion of $1 \times 10^{6} / \mathrm{kg}$ to $3 \times 10^{6} / \mathrm{kg}$ CAR T cells resulted in CR in 14 patients, while 12 patients were MRD-negative. Among the 12 MRD-negative CR patients, 10 received allo-HSCT at a median of 51 days after CAR $\mathrm{T}$ cell infusion; these patients remained leukemia-free. The remaining two patients were ineligible for allo-HSCT and developed CD19-negative relapse, indicating that the combination of CAR T-cell therapy and allo-HSCT improves long-term LFS. In a follow-up study of 51 B-ALL patients and two lymphoma patients, the 32 newlyrecruited patients received $1 \times 10^{6} / \mathrm{kg}$ CAR T cells along with lymphodepletion therapy comprised of fludarabine (flu) and cyclophosphamide (cy), or ifosfamide/etoposide [31]. Twenty-eight of the 53 patients achieved MRDnegative CR, with a median LFS of 18 months. Lymphodepletion with flu/cy significantly prolonged overall survival (OS). Twenty-one of the 28 MRD-negative CR patients received consolidative allo-HSCT at a median of 54 days after CAR T-cell infusion. Patients treated with alloHSCT exhibited significantly longer LFS (median LFS not reached) than the seven patients that did not receive alloHSCT (median LFS, 4.9 months). Additionally, researchers observed a shorter persistence of CD28-based KTE-C19 cells than 4-1BB-based CAR T cells, and hypothesized the difference might derive from CAR $\mathrm{T}$ cell exhaustion or immunological mechanisms. Albeit the limited persistence (less than 68 days) of CD28-based CAR T cell, it was sufficient to induce MRD-negative CR and served as an effective bridge to allo-HSCT.

Fifty-one $\mathrm{r} / \mathrm{r}$ B-ALL patients received $0.05 \times 10^{5} / \mathrm{kg}$ to $14 \times 10^{5} / \mathrm{kg}$ anti-CD19 CAR T cells at the Lu Daopei Hospital in China, and 20 patients received final-settled $1 \times$ $10^{5} / \mathrm{kg}$ CAR T cells. Forty-five patients achieved CR or CR with incomplete count recovery (CRi) [26]. Twenty-seven responding patients received consolidative allo-HSCT at a median of 84 days after CAR T-cell infusion. Twelve of these patients had complex chromosomal aberrations, 13 had adverse gene mutations (e.g., IKZF1 and TP53), and nine had extramedullary diseases (EMD), including central nervous system leukemia, testicular leukemia, diffused EMDs and others. All patients received myeloablative 


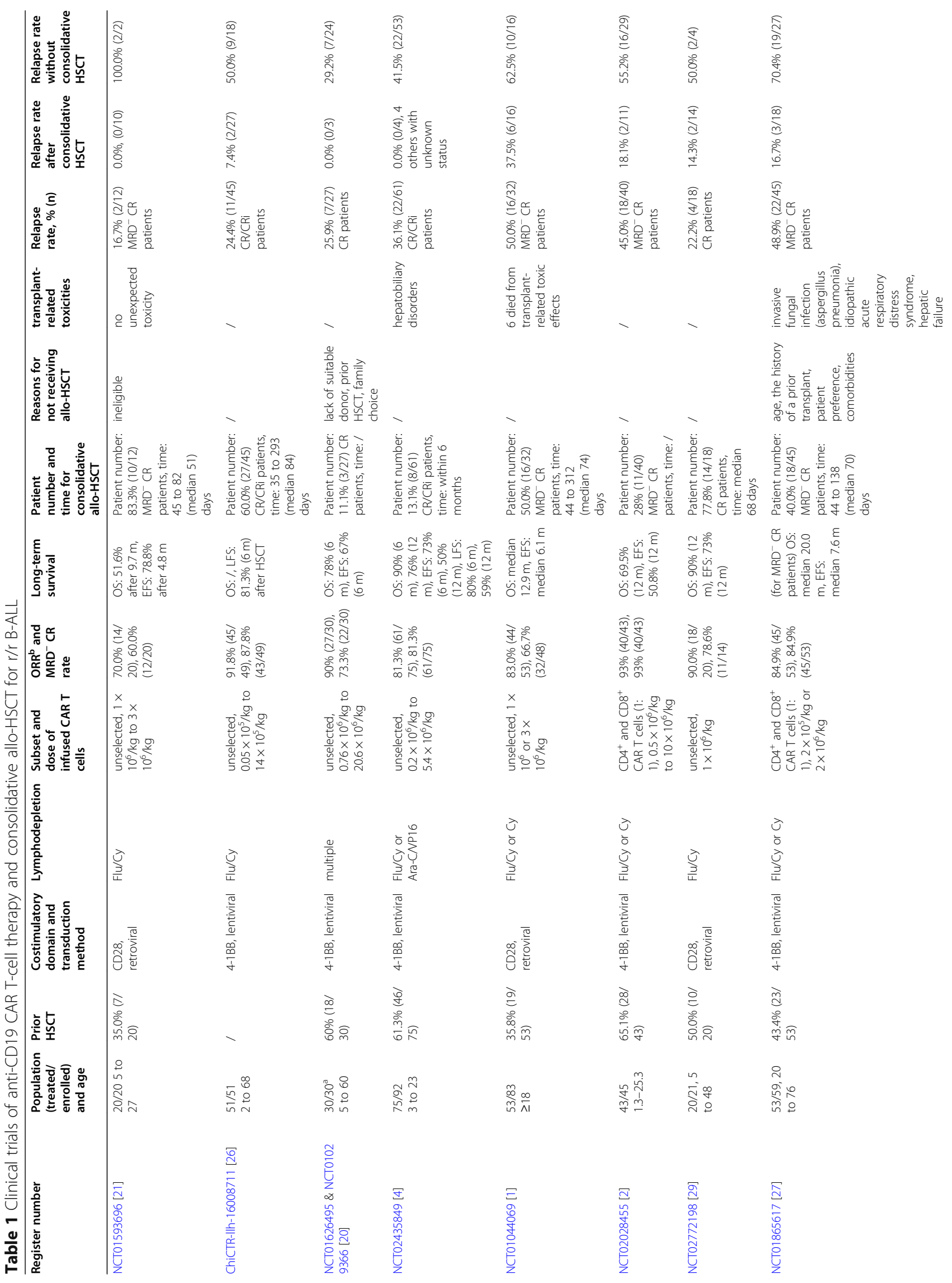




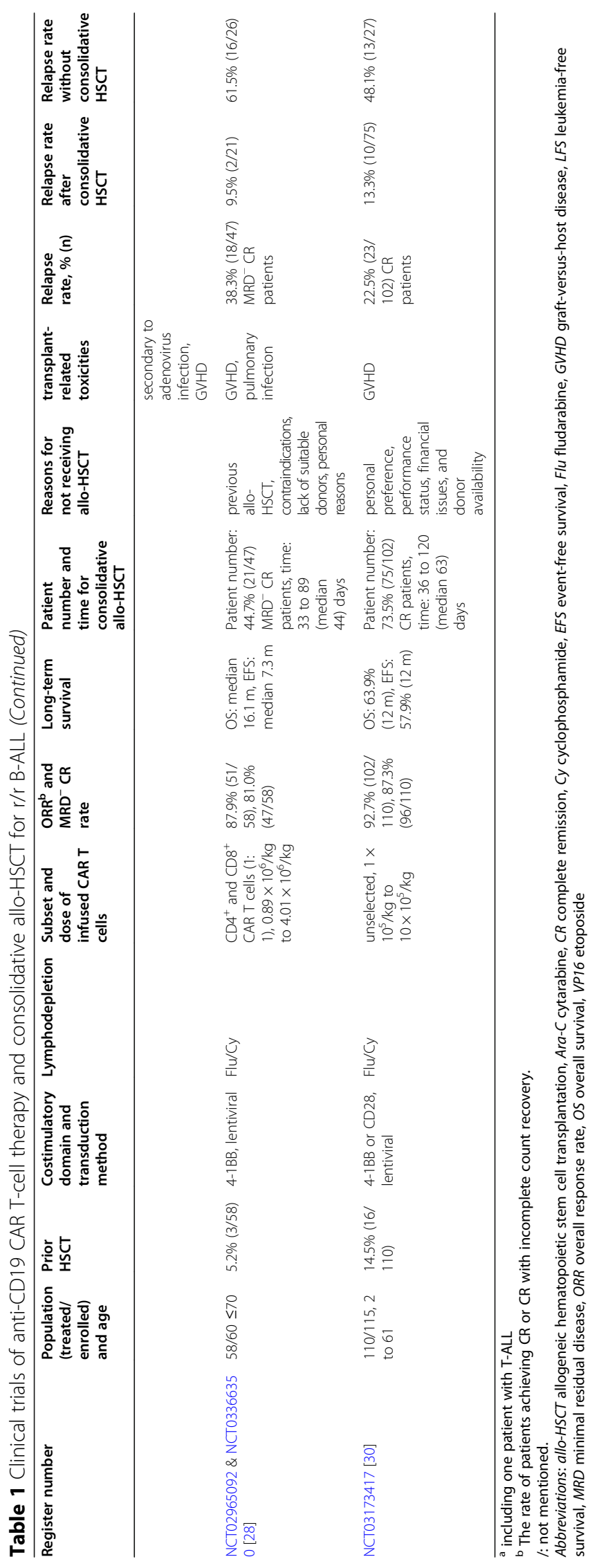


conditioning chemotherapy before transplantation of grafts from haploidentical donors, matched unrelated donors, or matched sibling donors. Of the 27 transplanted patients, 23 maintained MRD-negative CR (median of 133 days after allo-HSCT), two died of treatment-related mortalities (TRM), and two relapsed. The remaining 18 responding patients refused allo-HSCT, and nine of them relapsed. After adjusting for a uniform time point (90 days after CAR T-cell infusion) to eliminate the influence of early relapse, which precludes subsequent allo-HSCT, researchers found a significant difference in LFS between transplanted and non-transplanted patients. Overall, the study firstly indicated that consolidative allo-HSCT further reduced the relapse rate of $\mathrm{r} / \mathrm{r}$ B-ALL patients with high-risk gene mutations.

In the phase $1 / 2 \mathrm{a}$ clinical trial conducted at the Children's Hospital of Philadelphia and Hospital of the University of Pennsylvania, $30 \mathrm{r} / \mathrm{r}$ ALL patients received $0.76 \times 10^{6} / \mathrm{kg}$ to $20.6 \times 10^{6} / \mathrm{kg}$ CTL019 (tisagenlecleucel) cells and 27 achieved CR (22 were MRD-negative) [20]. Three patients received subsequent allo-HSCT and remained in CR for up to 12 months after CTL019 infusion, demonstrating the feasibility of allo-HSCT as consolidation treatment. Moreover, a correlation was observed between sustained remission and prolonged persistence (over three months) of CTL019 and B-cell aplasia (BCA), as the two patients exhibiting loss of CTL019 and recovery of normal B cells subsequently developed CD19-positive relapse. Therefore, researchers suggested that the sign of normal B cell return could potentially provide a window for consolidative allo-HSCT.

Subsequently, a phase 2, single-cohort, multicenter clinical trial of tisagenlecleucel was carried out with 75 $\mathrm{r} / \mathrm{r}$ B-ALL patients [4]. Infusion of $0.2 \times 10^{6} / \mathrm{kg}$ to $5.4 \times$ $10^{6} / \mathrm{kg}$ CAR T-cells resulted in CR or CRi in 61 patients, all of whom were MRD-negative. The median persistence of tisagenlecleucel in the blood was 168 days at data cut-off, with an ongoing persistence for up to 20 months. All responding patients experienced $\mathrm{BCA}$, and the probability of BCA at six months after CAR T-cell infusion was $83 \%$. Consolidative allo-HSCT was not taken preferentially due to the durable persistence of tisagenlecleucel, which indicated a high probability of cure. Only eight patients underwent allo-HSCT while in remission, two of whom developed MRD-positive disease, and two exhibited B cell recovery within six months of CAR Tcell infusion. All eight transplanted patients remained alive until study endpoint; four of these patients were still in remission. The relapse-free survival (RFS), eventfree survival (EFS), and OS were 80,73 , and $90 \%$ at six months, and 59,50 , and $76 \%$ at 12 months, suggesting the high probability of durable remission after treatment with tisagenlecleucel alone. Therefore, researchers suggested consolidative allo-HSCT only in patients with signs of relapse, such as MRD recurrence and B cell recovery.

In the phase 1 study conducted in Memorial Sloan Kettering Cancer Center, $16 \mathrm{r} / \mathrm{r}$ B-ALL patients received $3 \times 10^{6} / \mathrm{kg} 19-28 \mathrm{z}$ CAR T cells, resulting in complete molecular remission (CRm) in 12 patients [19]. Two patients refused allo-HSCT, two patients had pre-existing medical contraindications to allo-HSCT, and one patient was under evaluation for allo-HSCT; the remaining 7 $\mathrm{CRm}$ patients received consolidative allo-HSCT. At the follow-up of 2 to 24 months, no patients showed relapse after allo-HSCT, although two patients experienced severe transplantation-related complications and eventually died. Overall, the trial showed that $19-28 z$ CAR Tcell therapy provides an effective bridge for patients otherwise either ineligible or eligible but with MRD to receive standard of care allo-HSCT. A follow-up study in 53 patients and with a longer follow-up (median, 29 months) evaluated the efficacy of CAR $\mathrm{T}$ cells at a dose of $1 \times 10^{6} / \mathrm{kg}$ or $3 \times 10^{6} / \mathrm{kg}$ [1]. Sixteen of the $32 \mathrm{MRD}$ negative CR patients received allo-HSCT at a median of 74 days after 19-28z CAR T-cell infusion, six of whom relapsed. The remaining $16 \mathrm{MRD}$-negative $\mathrm{CR}$ patients did not receive allo-HSCT, and 10 of them relapsed. No significant difference in OS and EFS was observed between these two groups, indicating that consolidative allo-HSCT did not improve long-term outcomes. It is worth noting that a higher ratio of peak CAR T-cell expansion to baseline tumor burden (rather than the absolute magnitude of $\mathrm{T}$ cell expansion or disease burden) was associated with prolonged OS and EFS.

Forty-five pediatric and young adult $\mathrm{r} / \mathrm{r}$ B-ALL patients were enrolled in a phase 1 clinical trial of SCRICAR19v1 conducted in Seattle Children's Research Institute [2]. The infused CAR T cells comprised of $\mathrm{CD} 4^{+}$ and $\mathrm{CD} 8^{+}$CAR $\mathrm{T}$ cells at the ratio of $1: 1$, and the total dose was $0.5 \times 10^{6} / \mathrm{kg}$ to $10 \times 10^{6} / \mathrm{kg}$. Eleven of the 40 MRD-negative CR patients proceeded to allo-HSCT (two developed CD19-positive relapse), and the remained 29 patients received no consolidative alloHSCT (16 experienced leukemia recurrence). Researchers found that the early loss of functional CAR $\mathrm{T}$ cells reflected by the short duration of BCA (median duration of three months), significantly increased the risk of CD19-positive relapse. Additionally, flu/cy lymphodepletion prolonged the persistence of CAR T cells. Further analysis revealed that functional CAR T cell persistence for more than six months was a critical determinant of durable remission [32]. Furthermore, some $\mathrm{T}$ cell-intrinsic features, including elevated expression of LAG3 and reduced secretion of TNF- $\alpha$ in circulating $\mathrm{CD}^{+} \mathrm{T}$ cells at the time of leukapheresis, predicted therapeutic failure and early relapse. Therefore, these features can serve as indicators for consolidation 
therapies. Data from a phase $1 / 2$ trial with a follow-up time of more than one year were further analyzed to evaluate the effect of allo-HSCT on long-term outcomes [23]. Fifty of the 64 enrolled patients achieved CR, 32 of whom received the phase 1 dose and 18 received the phase 2 dose of $1 \times 10^{6} / \mathrm{kg}$ CAR T cells. Seventeen CR patients had no history of allo-HSCT and exhibited prolonged LFS after treatment with consolidative alloHSCT following CAR T-cell therapy. However, there was no significant benefit of consolidative allo-HSCT in patients previously treated with allo-HSCT. Interestingly, a certain benefit of consolidative allo-HSCT was observed in $\mathrm{CR}$ patients who were at high risk of relapse with an early loss of BCA (before 63 days), regardless of allo-HSCT history. These findings suggest that patients should be considered for consolidative allo-HSCT during remission after CAR T-cell therapy if they have no history of allo-HSCT, or if they had a history of allo-HSCT but with a short persistence of functional CAR T cells.

A phase $1 \mathrm{~b} / 2$ clinical trial of CD28-based anti-CD19 CAR T-cell therapy was conducted at Sheba Medical Center in Israel [29]. Twenty $\mathrm{r} / \mathrm{r}$ ALL patients received CAR T-cell infusion $\left(1 \times 10^{6} / \mathrm{kg}\right)$. Ten of these patients had a history of allogeneic or haploidentical HSCT, and eight of them had active resistant extramedullary (EM) leukemic involvement at recruitment. The active EM sites included the kidney, spine, femur, temporal lobe, leptomeningeal enhancement, cerebrospinal fluid, paraspinal, scalp, and diffuse bone infiltration. Eighteen patients achieved $\mathrm{CR}$, and 14 of them were referred to consolidative allo-HSCT within a median of 68 days after CAR T-cell infusion. Of these 14 patients, seven had prior transplantation, and five had a history of active EM sites. No severe transplantation-related toxicities were reported. Two of the 14 transplanted patients exhibited CD19-positive relapse, and two of the four nontransplanted patients had CD19-positive and CD19negative relapse, respectively. The findings of the study demonstrated the safety of consolidative allo-HSCT after CAR T-cell therapy in patients with prior transplantation or EM leukemia and provided preliminary evidence that consolidative allo-HSCT reduces the relapse rate.

In a phase $1 / 2$ clinical trial conducted at the Fred Hutchinson Cancer Research Center, 53 B-ALL patients received $2 \times 10^{5} / \mathrm{kg}$ or $2 \times 10^{6} / \mathrm{kg}$ anti-CD19 CAR T cells comprised of $\mathrm{CD}^{+}$and $\mathrm{CD} 8^{+}$cells at a 1:1 ratio [27]. Forty-five patients achieved MRD-negative CR. High throughput sequencing revealed that the absence of the malignant clone in the bone marrow three weeks after CAR T-cell infusion was associated with prolonged EFS in patients with MRD-negative CR. Furthermore, stepwise multivariable modeling demonstrated that low prelymphodepletion lactate dehydrogenase (LDH) $(\leq 210 \mathrm{U} /$
L), high pre-lymphodepletion platelet count $(\geq 100,000$ / $\mu \mathrm{L}$ ), and incorporation of fludarabine into the lymphodepletion regimen were independent prognostic factors of improved EFS in patients with MRD-negative CR. Based on age, history of transplantation, patient preference, and comorbidities, 18 MRD-negative CR patients received consolidative allo-HSCT, and three of these patients experienced CD19-positive relapse. Four patients died due to transplantation-related adverse effects, including invasive fungal infection (aspergillosis), idiopathic acute respiratory distress syndrome, hepatic failure secondary to adenovirus infection, and GVHD. Multivariate analysis revealed that consolidative allo-HSCT was associated with prolonged EFS. Interestingly, a longer time between CAR T-cell infusion and allo-HSCT ( $\geq 80$ days) was associated with a higher risk of death and increased non-relapse mortality [33]. Therefore, researchers concluded that both high-risk and low-risk patients might benefit from allo-HSCT and suggested that eligible adult MRD-negative CR B-ALL patients should undergo allo-HSCT as soon as possible after anti-CD19 CAR T-cell therapy.

In a non-randomized interventional pragmatic clinical trial conducted at our institution, $58 \mathrm{r} / \mathrm{r}$ B-ALL patients received $0.89 \times 10^{6} / \mathrm{kg}$ to $4.01 \times 10^{6} / \mathrm{kg}$ CAR T cells comprised of $\mathrm{CD}^{+}$and $\mathrm{CD} 8^{+} \mathrm{T}$ cells at a ratio of 1:1 [28]. Forty-seven of the 58 patients achieved MRD-negative CR. Subsequently, 21 MRD-negative CR patients received consolidative allo-HSCT within three months after CAR T-cell infusion. Patients received peripheral blood stem cell transplantation with or without bone marrow transplantation, with grafts from matched sibling donors or haploidentical donors. Additionally, patients received myeloablative conditioning chemotherapy and cyclosporine/tacrolimus, methotrexate, mycophenolate mofetil, anti-CD25 monoclonal antibody, or anti-thymocyte globulin to prevent GVHD. At a median follow-up time of 10.8 months after CAR T-cell infusion, two patients experienced TRM and two relapsed after consolidative allo-HSCT. Twenty-six MRD-negative $\mathrm{CR}$ patients did not receive consolidative allo-HSCT for various reasons, and 16 of them experienced recurrence. EFS and RFS (but not OS) differed significantly between transplanted and non-transplanted patients. Notably, subgroup analyses indicated improved EFS and RFS in patients with high $(\geq 5 \%)$ pre-infusion bone marrow MRD assessed by flow cytometry, or poor prognostic markers, including complex karyotypes, BCR/ABL1, MLL/AF4, TP53, and $E 2 A / P B X 1$ mutations.

In the recent published phase $1 / 2$ clinical trial conducted at the Lu Daopei Hospital in China, 110 pediatric and adult patients received fludarabine and cyclophosphamide lymphodepleting chemotherapy, and single anti-CD19 CAR T-cell infusion of $1 \times 10^{5} / \mathrm{kg}$ to $10 \times$ $10^{5} / \mathrm{kg}$ [30]. Patients were with high-risk factors, including EMDs, BCR-ABL, TEL-AML1, E2A-PBX1, E2A-HLF 
fusion genes, IKZF1, TP53 gene mutations, and posttransplant relapse. Morphologic CR was achieved in $92.7 \%$ patients, and MRD-negative CR in $87.3 \%$ patients. Of the $102 \mathrm{CR}$ patients, 75 received consolidative allo-HSCT at a median of 63 days (range, 36-120 days) after CAR T-cell infusion. Patients received conventional myeloablative conditioning regimens, and grafts from HLA-identical sibling donors, matched-unrelated donors, or haploidentical donors, and GVHD prophylaxis comprised of cyclosporine, methotrexate, and mycophenolate mofetil. The 1-year OS and LFS were 79.1 and $76.9 \%$ for patients bridging into allo-HSCT, and 32 and $11.6 \%$ for patients receiving CAR T-cell therapy alone. Multivariable analysis indicated significantly improved OS and LFS in patients with consolidative allo-HSCT. These findings further demonstrated the benefit of consolidative allo-HSCT in patients with pre-treatment high-risk factors.

Taking into consideration that data from the above clinical trials, a total of $429 \mathrm{r} / \mathrm{r}$ B-ALL patients achieved CR/CRi after anti-CD19 CAR T-cell therapy. Of these responding patients, 203 proceeded to consolidative alloHSCT, of whom only 27 (13.3\%) relapsed. On the other hand, relapse was observed in 116 (51.3\%) of the 226 patients who did not receive consolidative allo-HSCT. Because of differences in CAR design, trial protocol, and patient characteristics, no conclusions can be drawn with confidence; nevertheless, consolidative allo-HSCT seems to reduce the recurrence rate. The time window for consolidative allo-HSCT ranged from 33 days to 312 days after CAR T-cell infusion, and the median time was 44 days to 84 days after CAR T-cell infusion. The most common transplantation-related toxicities were GVHD, infections, and hepatic or respiratory dysfunctions. The main reasons for not providing consolidative allo-HSCT included a suboptimal response to CAR T-cell therapy, age, comorbidities, contraindications, prior HSCT, lack of suitable donor, family choice, and patient preference. Altogether, the findings of these studies suggest that consolidative allo-HSCT may improve long-term outcomes for most r/r B-ALL patients (Table 1).

In addition to the above published studies, there are several clinical trials (NCT03110640, ChiCTR1800017669 ... ...) ongoing in domestic clinical centers. Despite the bridging strategy is more and more popular among domestic clinical centers, large-scale multicenter clinical trial is still lacked. It is meaningful to conduct a multicenter clinical trial with uniform standards of both CAR T-cell manufacture and patients' management.

\section{Factors associated with consolidative Allo-HSCT benefit}

By reviewing available data from the literature, we identified several risk factors associated with relapse after CAR T-cell therapy. These factors can be classified into the following categories: (1) pre-treatment (baseline) patient characteristics, such as complex karyotypes or adverse genes, high pre-infusion MRD by flow cytometry in the bone marrow, high pre-lymphodepletion $\mathrm{LDH}$, and low pre-lymphodepletion platelet count; (2) lymphodepletion regimen without fludarabine; (3) post-CAR Tcell infusion parameters, such as persistent leukemic cells in the bone marrow and early loss of CAR T-cells.

\section{Pre-treatment patient characteristics}

Complex karyotypes and overexpression of certain genes, including $M L L / A F 4, B C R / A B L 1, E 2 A / P B X 1$, and TP53, have been associated with an increased risk of relapse and poor prognosis in $\mathrm{r} / \mathrm{r}$ B-ALL [34-37]. Numerous studies reported that $M L L$-rearranged, $B C R / A B L 1^{+}$, and TCF3ZNF384 $^{+}$B-ALL patients were associated with an increased risk of CD19-negative myeloid lineage relapse after CD19-targeted immunotherapy, such as CAR T-cell therapy and blinatumomab [38-41]. The lineage switch can lead to loss of CD19 and resistance to CD19-targeted CAR T-cell therapy. Therefore, patients with chromosomal alterations or high-risk genes should be considered for standard of care allo-HSCT, and CAR T-cell therapy can serve as a suitable bridge to turn related genes negative and transfer patients to allo-HSCT [26, 42]. Furthermore, we have previously shown that patients with poor prognostic markers would benefit from the early consolidative allo-HSCT after CAR T-cell therapy [28].

Many clinical trials showed that patients with high pre-infusion CD19-positive leukemia burden had low levels of anti-CD19 CAR T cells, increasing the probability of post-CAR T-cell therapy relapse $[1,28,43]$. The decrease in surface CAR expression may result from receptor internalization induced by exposure to CD19positive leukemic cells [44]. Moreover, a high risk of relapse is observed in patients with high pre-infusion leukemia burden, even in those showing robust CAR $\mathrm{T}$ cell expansion. A high ratio of peak CAR $T$ cell expansion to leukemia burden $(>1)$ was found to predict longer EFS and OS, suggesting that an optimal ratio of CAR $\mathrm{T}$ cells to CD19-positive leukemia cells is more probable in patients with a low leukemia burden than in those with a high leukemia burden, despite the lower CAR T cell expansion in patients with a low leukemia burden [1]. Intriguingly, we have previously shown that in patients with high leukemia burden (pre-infusion bone marrow MRD $\geq 5 \%$ as assessed by flow cytometry), consolidative allo-HSCT after CAR T-cell therapy significantly prolonged EFS and RFS [28].

Although high pre-lymphodepletion LDH level $(>210$ $\mathrm{U} / \mathrm{L})$ and low platelet count $(<100,000 / \mu \mathrm{L})$ showed little association with bone marrow leukemia burden, they were strongly correlated with the need of systemic bridging chemotherapy between leukapheresis and 
lymphodepletion [27]. Besides, high LDH levels and low platelet count were more frequent in patients with cytogenetic alterations and those with extramedullary diseases, respectively. Stepwise multivariate modeling revealed that normal pre-lymphodepletion LDH $(\leq 210 \mathrm{U} / \mathrm{L})$ and platelet count $(\geq 100,000 / \mu \mathrm{L})$ were independent predictors of prolonged EFS in patients exhibiting MRD-negative CR. Other studies also indicated that serum LDH levels were related to an immunosuppressive microenvironment, tumor burden, and proliferative activity in B-cell and other malignancies [45-50]. Hence, high pre-lymphodepletion LDH concentration and low pre-lymphodepletion platelet count can reflect not only leukemia burden, but also an aggressive disease phenotype, making them potential high-risk indicators of post-CAR T-cell relapse.

\section{Lymphodepletion regimen}

Several clinical studies indicated that the incorporation of fludarabine to the lymphodepletion regimen could improve the expansion and persistence of CAR T cells, rate and depth of response, as well as OS and RFS, in $r / r$ B-ALL and other B-cell malignancies [2, 3, 27, 31, 51]. Delay or abrogation of an immune response against the murine single-chain variable fragment component of the CAR, increased levels of cytokines that support $\mathrm{T}$ cell proliferation and survival, and IDO downregulation in tumor cells may contribute to the enhanced antitumor activity of CAR $\mathrm{T}$ cells after strong lymphodepletion [3, 51, 52]. Therefore, patients that did not receive fludarabine are at high risk of relapse and may require consolidative allo-HSCT to achieve sustained remission.

\section{Post-infusion monitoring}

The depth of remission determined by marrow leukemic index clones for IGH, IGK, TRB, TRD, and TRG (detected by high throughput sequencing) has been associated with favorable long-term outcomes after CAR Tcell therapy [27]. Studies indicated that the absence of leukemic clones three weeks after CAR T-cell infusion in MRD-negative CR patients was associated with prolonged EFS and OS. Therefore, patients with persistent leukemic clones should be recommended for consolidative allo-HSCT to deepen remission and reestablish hematopoietic system and immune system.

A low peak and a short persistence of CAR $\mathrm{T}$ cells have also been associated with CD19-positive relapse, and most patients with CD19-positive relapse had either undetectable or very low $(<30$ copies/ $\mu$ g DNA) CAR T cell counts before or at the time of relapse [27, 28]. Additionally, the persistence of functional CAR $\mathrm{T}$ cells for more than six months was a critical determinant of durable remission [32]. Furthermore, as the presence of BCA indicates sustained immunosurveillance of functional CAR $\mathrm{T}$ cells, a short duration of BCA (less than three months) was identified as a factor associated with an increased risk of CD19-positive relapse, and the median time from loss of BCA to CD19-positive relapse was 3.7 months [2, 20, 27]. Encouragingly, the MRDnegative CR patients who developed recovery of normal $\mathrm{B}$ cells within 63 days after CAR $\mathrm{T}$ cell infusion could obtain a certain benefit from consolidative allo-HSCT [23]. Therefore, the recovery of normal B cells can be regarded as an indicator of following CD19-positive relapse and can potentially provide a window for consolidative allo-HSCT.

Overall, relapse after CAR T-cell therapy can be potentially predicted by the aforementioned parameters, which exist during the whole procedure of CAR T-cell therapy, including screening, lymphodepletion, and follow-up. The prior existence or newly occurrence of these factors may facilitate timely treatment with consolidative allo-HSCT, improving disease outcomes.

\section{Therapeutic window and consolidative Allo-HSCT regimen}

The median time for consolidative allo-HSCT in most centers ranged from 44 days to 84 days after CAR $\mathrm{T}$-cell infusion (Table 1), before or immediately at the occurrence of high-risk factors. A long-term follow-up study indicated that late consolidative allo-HSCT (more than 80 days after CAR T-cell infusion) significantly increased the risk of death [33]. A recent published retrospective study indicated a lower incidence of relapse and a higher 2-year LFS in pre-transplant MRD-negative patients than in pre-transplant MRD-positive patients [53]. Besides, the relapse rate of MRD-negative $C R$ patients was approximately $10 \%$ three months after CAR T-cell therapy $[1,21,28]$. These findings support the usefulness of early transplantation discussion and HLA typing for suitable B-ALL patients before the administration of CAR $\mathrm{T}$-cell therapy, and we recommend treatment with consolidative allo-HSCT within three months of CAR T-cell therapy to maximize its benefit and minimize the risk of toxicity-related death.

As prior anti-CD19 CAR T-cell therapy can modulate the immune microenvironment and cause endothelial damage, the therapeutic protocol for consolidative alloHSCT may need to be re-customized [54-56]. The donor types, graft cell types, conditioning therapies, and GVHD prophylaxis varied among several large-scale studies (Table 2). Overall, the protocols were similar to conventional allo-HSCT in $\mathrm{r} / \mathrm{r}$ B-ALL. A recent study with a median follow-up of 36 months revealed no possible toxicity that was disproportionately more common among patients receiving consolidative allo-HSCT than in those undergoing allo-HSCT without prior CAR Tcell therapy [33]. Although there are no studies comparing different consolidative allo-HSCT protocols, available 
preliminary data suggest that conventional protocols are tolerable and feasible in most cases. Furthermore, researchers found that CAR T-cell therapy followed by CD34-selected T-cell depleted allo-HSCT was associated with lower incidence of TRM and prolonged OS when compared with unmodified allo-HSCT [57].

\section{Necessity of consolidative Allo-HSCT}

Despite the potential benefit and safety of consolidative allo-HSCT after CAR T-cell therapy, not every patient should undergo allo-HSCT considering cost effectiveness and other potential risks. Factors, such as age, prior transplantation, CAR T-cell design, and treatment protocol should be taken into account in the decision making for consolidative allo-HSCT.

While adult $\mathrm{r} / \mathrm{r}$ B-ALL patients typically benefit from consolidative allo-HSCT, pediatric and young adult patients may achieve long-term remission without consolidative allo-HSCT, with the 12-month EFS of approximately $50 \%$ when only a quarter patients were referred to allo-HSCT [2, 4]. Apart from differences in the mechanisms underlying leukemia development, the superior performance of pediatric patients might derive from a better quality of isolated $\mathrm{T}$ cells, as adult $\mathrm{r} / \mathrm{r} \mathrm{B}-$ ALL patients were probably heavily-treated [58].

Generally, patients undergoing allo-HSCT before CAR $\mathrm{T}$-cell therapy are precluded from consolidative alloHSCT, since second or third allo-HSCT has been associated with high risks of TRM [20,27, 28]. Despite this, some patients still underwent second allo-HSCT after donor-derived CAR T-cell therapy. In the Sleeping Beauty-engineered donor-derived CAR T-cell therapy for B-ALL patients relapsed after allo-HSCT, 2 of the 6 MRD-negative CR patients received consolidative alloHSCT and remained leukemia-free, the other 4 did not receive second allo-HSCT and 2 relapsed with CD19positive disease [59]. In the SCRI-CAR19v1 study, 10 of 33 patients with a history of allo-HSCT received second allo-HSCT after CAR T-cell therapy-mediated CR, and five patients showed long-term remission [23]. Of the 23 patients who did not receive second allo-HSCT, eight patients showed long-term remission. Although the role of consolidative allo-HSCT was not clear in patients previously treated with allo-HSCT, these patients can still benefit when with short persistence of functional SCRICAR19v1. Therefore, a decision regarding a second alloHSCT after donor-derived CAR T-cell therapy should be made on a case-by-case basis.

Preclinical studies indicated that CAR T cells with CD28 costimulatory domain had an increased expression of exhaustion-related genes, resulting in a relatively short duration after infusion, despite robust expansion and good efficacy at early time [60,61]. Consistently, CD28-based antiCD19 CAR T cells exhibited a shorter persistence than 41BB-based anti-CD19 CAR T cells [1, 18-21, 29, 62]. Nevertheless, treatment strategies and outcomes of CD28based CAR T-cell therapies varied significantly among different studies. Most patients at the National Cancer Institute and Sheba Medical Center underwent consolidative allo-HSCT and exhibited improved survival, whereas only half of MRD-negative CR patients received consolidative allo-HSCT at the Memorial Sloan Kettering Cancer Center, and no clinical benefit was observed [1, 21, 29]. At present, there is insufficient evidence to conclude whether CD28based CAR T-cell therapy should be consolidated with subsequent allo-HSCT.

As novel technologies came up, the necessity of consolidative allo-HSCT should be discussed extensively. In a humanized anti-CD19 CAR T-cell study, no survival benefit was observed in patients receiving consolidative allo-

Table 2 Consolidative allo-HSCT regimen

\begin{tabular}{|c|c|c|c|c|}
\hline Register number & Donor type & Cell type & Conditioning therapy & GVHD prophylaxis \\
\hline ChiCTR-IIh-16008711 [26] & $\begin{array}{l}17 \text { Haploidentical } \\
7 \text { MUD } \\
3 \text { MSD }\end{array}$ & / & $\begin{array}{l}27 \mathrm{MAC}(24 \mathrm{TBI} / \mathrm{CY} / \mathrm{Ara}-\mathrm{C} / \mathrm{MeCCNU} / \pm \mathrm{ATG}, \\
3 \mathrm{Bu} / \mathrm{CY} / \mathrm{Ara}-\mathrm{C} / \mathrm{MeCCNU} / \pm \mathrm{ATG})\end{array}$ & / \\
\hline NCT01865617 [27] & $\begin{array}{l}3 \text { MRD } \\
9 \text { MUD } \\
1 \text { mMURD } \\
1 \text { Haploidentical } \\
5 \text { UCT }\end{array}$ & $\begin{array}{l}13 \text { PBSC } \\
1 \text { BM } \\
5 \text { Cord }\end{array}$ & $\begin{array}{l}14 \text { MAC } \\
2 \text { RIC } \\
3 \text { NMA }\end{array}$ & $\begin{array}{l}5 \mathrm{CNI}+\mathrm{MMF} \\
1 \mathrm{CNI}+\mathrm{MMF}+\text { sirolimus } \\
9 \mathrm{CNI}+\mathrm{MTX} \\
3 \mathrm{CNI}+\mathrm{MTX}+\text { abatacept } \\
1 \mathrm{CNI}+\mathrm{MMF}+\text { PtCy }\end{array}$ \\
\hline NCT02965092 \& NCT03366350 [28] & $\begin{array}{l}8 \mathrm{MSD} \\
13 \text { haploidentical }\end{array}$ & $\begin{array}{l}16 \text { PBSC } \\
5 \text { PBSC+BM }\end{array}$ & $21 \mathrm{MAC}(\mathrm{VP} 16+\mathrm{BUCY})$ & $\begin{array}{l}8 \text { CsA/FK506 + MTX } \\
13 \text { FK506 + MTX + MMF + } \\
\text { CD25 antibody+ATG }\end{array}$ \\
\hline NCT03173417 [30] & $\begin{array}{l}16 \text { MSD } \\
9 \text { MUD } \\
50 \text { Haploidentical }\end{array}$ & / & $\begin{array}{l}75 \text { MAC ( } 69 \text { TBI-based for }>5 y \text { patients, } \\
6 \text { Bu-based for } \leq 5 y \text { patients) }\end{array}$ & $75 \mathrm{Cs} A+M T X+M M F$ \\
\hline
\end{tabular}

Ara-C cytarabine, ATG anti-thymocyte globulin, BM bone marrow, Bu Busulfan, BUCY busulfan and cyclophosphamide, CNI calcineurin inhibitor, CSA Cyclosporine, $\mathrm{CY}$ cyclophosphamide, FK506 tacrolimus, MAC myeloablative conditioning, MeCCNU methyl-CCNU-semustine, MMF mycophenolate mofetil, mMURD mismatched unrelated donor, MRD matched related donor, MSD matched sibling donor, MTX methotrexate, MUD matched unrelated donor, NMA nonmyeloablative conditioning, PBSC peripheral blood stem cell, PtCy posttransplant cyclophosphamide, RIC reduced-intensity conditioning, TBI total body irradiation, UCT umbilical cord transplant, VP16 etoposide 
HSCT [63]. Similarly, CAR designs or treatment strategies to reduce CD19-negative relapse or prolong the functional persistence of CAR T cells, such as sequential infusion of anti-CD19 and anti-CD22 CAR T cells and combination with PD-1 inhibitors, may provide long-term remission without consolidative allo-HSCT [64-68]. In particular, two patients received universal CAR $\mathrm{T}$ cells and all received consolidative allo-HSCT [69]. It raises the question of whether consolidative allo-HSCT is necessary after universal CAR T-cell therapy, as the CAR T cells would be eventually eliminated by immunological rejection [70].

Considering the high heterogeneity among and within patients, different CAR $\mathrm{T}$ cell products and continuous improved treatment strategies, the application of consolidative allo-HSCT cannot be simply generalized. However, consolidative allo-HSCT is strongly recommended for adult high-risk r/r B-ALL patients who have no history of allo-HSCT and achieve MRD-negative CR after murine anti-CD19 CAR T-cell therapy.

\section{Recommendation for the assessment and management of $r / r$ B-ALL patients in CAR T-cell therapy}

Based on the findings of clinical trials and our institutional experiences, we established a recommendation for the assessment and management of $\mathrm{r} / \mathrm{r}$ B-ALL patients undergoing anti-CD19 CAR T-cell therapy. (Fig. 1) High-risk patients should be considered for allo-HSCT, and suitable donors should be identified before CAR Tcell therapy, enabling the timely consolidative alloHSCT if eligible. Patients without risk factors may achieve long-term remission, with continuous expression of CAR-targeted tumor antigen and durable functional CAR T cells [71]. Therefore, regular assessment on primary disease, including bone marrow MRD, fusion genes and high risk cytogenetics, and treatment effect, including CAR T cell persistence and B cell recovery, would enable the prompt identification of patients at a high risk of relapse. Rise of MRD, occurrence of high-risk genes or chromosome mutations, decrease of CAR T cells, and recovery of normal B cells, can be used as indications for consolidative allo-HSCT. However, there are still limitations of this recommendation, as no cut-off value but a trend was given for high-risk factors. More clinical data are needed to determine the optimal cut-off values.

\section{Conclusions}

Consolidative allo-HSCT has been used in $\mathrm{r} / \mathrm{r}$ B-ALL patients after anti-CD19 CAR T-cell therapy in several clinical trials and showed acceptable safety and efficacy. In addition to anti-CD19 CAR T-cell therapy, anti-CD22 CAR T-cell therapy could also be consolidated with subsequent allo-HSCT, providing favorable EFS, LFS, and OS, as well as exhibiting a satisfactory safety profile [72-74]. Consolidative allo-HSCT could also be applied in patients with other B-cell malignancies, including B-cell non-Hodgkin lymphoma and chronic lymphocytic leukemia [33]. These clinical data indicate a demand for discussion of consolidative strategy in more other targeted CAR T therapies and other diseases.

Based on available clinical data, we recommend consolidative allo-HSCT in eligible high-risk patients to reduce the incidence of recurrence after CAR T-cell therapy and improve the quality of life. However, future randomized clinical trials and long-term outcome data are required to establish the clinical value of allo-HSCT after CAR T-cell therapy. The role of consolidative allo-

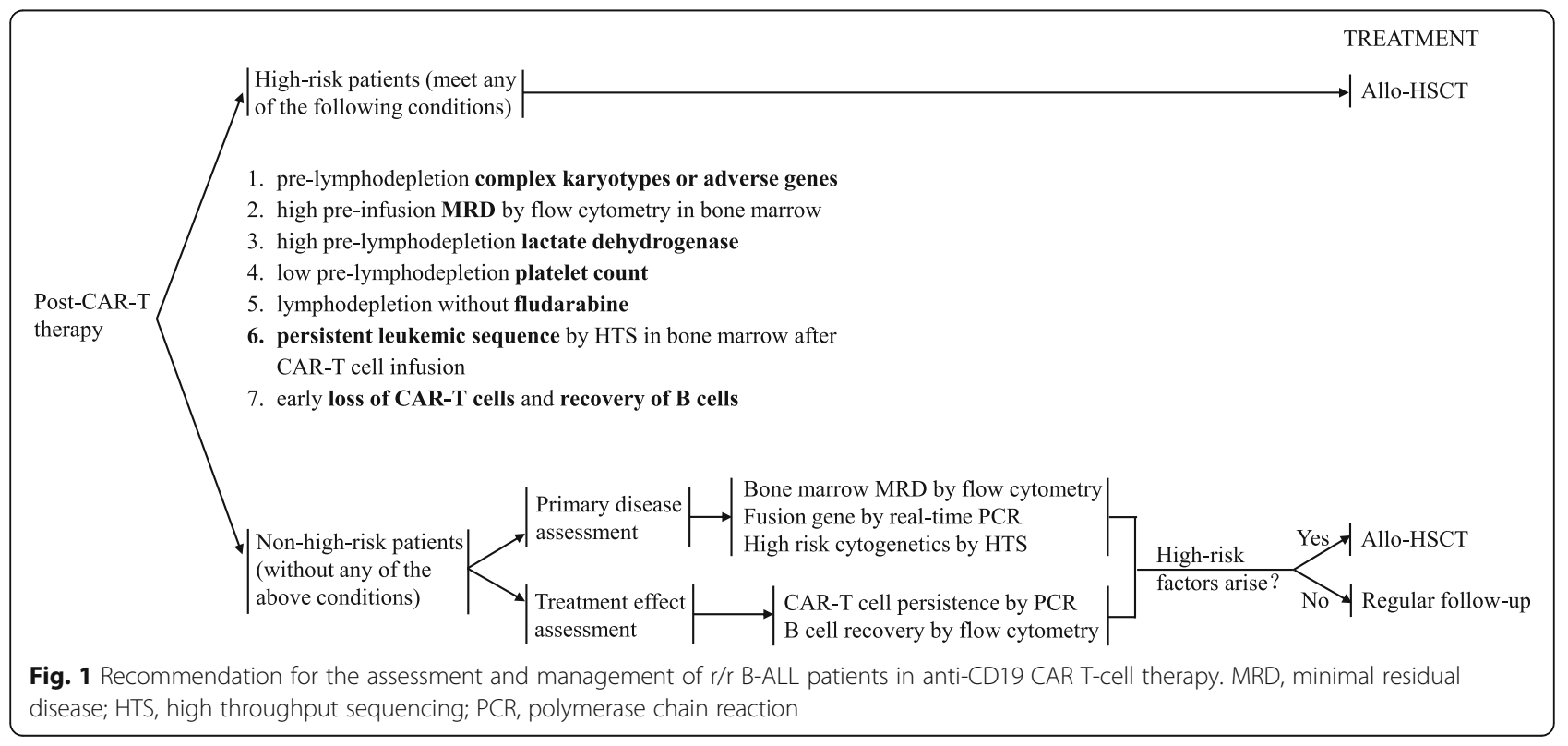


HSCT should be constantly redefined with the development of novel CAR designs and combination therapies.

\begin{abstract}
Abbreviations
Allo-HSCT: Allogeneic hematopoietic stem cell transplantation; BCA: B-cell aplasia; CAR T: Chimeric antigen receptor-modified T cell; CR: Complete remission; CRm: Complete molecular remissions; cy: Cyclophosphamide; EFS: Event-free survival; EMD: Extramedullary disease; flu: Fludarabine; GVHD: Graft-versus-host disease; HTS: High throughput sequencing; LDH: Lactate dehydrogenase; LFS: Leukemia-free survival; MRD: Minimal residual disease; OS: Overall survival; RFS: Relapse-free survival; r/r BALL: Relapsed/refractory B-cell acute lymphoblastic leukemia; TRM: Treatment-related mortalities
\end{abstract}

\section{Acknowledgements}

Not applicable.

\section{Authors' contributions}

$\mathrm{HM}$ and $\mathrm{YH}$ designed the study. $\mathrm{HJ}$ and $\mathrm{HM}$ wrote the initial drafts. HM and YH revised the review and finalized the last version of the article. All authors read and approved the final manuscript.

\section{Funding}

This study was supported by National Key R\&D Program of China 2019YFC1316203.

\section{Availability of data and materials}

Data sharing is not applicable to this article as no datasets were generated or analyzed during the current study.

\section{Ethics approval and consent to participate}

Not applicable.

\section{Consent for publication}

Not applicable.

\section{Competing interests}

The authors declare that they have no competing interests.

Received: 18 September 2020 Accepted: 5 November 2020 Published online: 25 November 2020

\section{References}

1. Park JH, Riviere I, Gonen M, et al. Long-term follow-up of CD19 CAR therapy in acute lymphoblastic leukemia. N Engl J Med. Feb 2018;378(5):449-59.

2. Gardner RA, Finney O, Annesley C, et al. Intent-to-treat leukemia remission by CD19 CAR T cells of defined formulation and dose in children and young adults. Blood. 2017;129(25):3322-31.

3. Turtle CJ, Hanafi LA, Berger C, et al. CD19 CAR-T cells of defined CD4(+): CD8(+) composition in adult B cell ALL patients. J Clin Investig. Jun 2016; 126(6):2123-38.

4. Maude SL, Laetsch TW, Buechner J, et al. Tisagenlecleucel in children and young adults with B-cell lymphoblastic leukemia. N Engl J Med. Feb 2018; 378(5):439-48.

5. Porter D, Frey N, Wood PA, Weng YQ, Grupp SA. Grading of cytokine release syndrome associated with the CAR T cell therapy tisagenlecleucel. J Hematol Oncol. 2018;11:12.

6. Prasad V. Tisagenlecleucel - the first approved CAR-T-cell therapy: implications for payers and policy makers. Nat Rev Clin Oncol. 2018;15(1):11.

7. Zhang LN, Song YP, Liu DL. CD19 CAR-T cell therapy for relapsed / refractory acute lymphoblastic leukemia: factors affecting toxicities and long-term efficacies. J Hematol Oncol. 2018;11:5.

8. Wang J, Hu Y, Huang H. Acute lymphoblastic leukemia relapse after CD19targeted chimeric antigen receptor T cell therapy. J Leukoc Biol. Dec 2017; 102(6):1347-56.

9. Cheng $\mathrm{LL}$, Zhao $\mathrm{L}$, Zhang $\mathrm{YY}$, et al. Understanding the mechanisms of resistance to CAR T-cell therapy in malignancies. Front Oncol 2019;9:9.

10. Pulsipher MA. Are CAR T cells better than antibody or HCT therapy in BALL? Hematol Am Soc Hematol Educ Prog. Nov 2018:16-24.
11. Balduzzi A, De Lorenzo P, Schrauder A, et al. Eligibility for allogeneic transplantation in very high risk childhood acute lymphoblastic leukemia: the impact of the waiting time. Haematologica. 2008;93(6):925-9.

12. Zhao XS, Liu YR, Xu LP, et al. Minimal residual disease status determined by multiparametric flow cytometry pretransplantation predicts the outcome of patients with ALL receiving unmanipulated haploidentical allografts. Am J Hematol. May 2019;94(5):512-21.

13. Gao C, Liu SG, Yue ZX, et al. Clinical-biological characteristics and treatment outcomes of pediatric pro-B ALL patients enrolled in BCH-2003 and CCLG-2008 protocol: a study of 121 Chinese children. Cancer Cell Int. Nov 2019;19(1):9.

14. Eckert $\mathrm{C}$, Biondi $\mathrm{A}$, Seeger $\mathrm{K}$, et al. Prognostic value of minimal residual disease in relapsed childhood acute lymphoblastic leukaemia. Lancet. 2001; 358(9289):1239-41.

15. Wood B, Wu D, Crossley B, et al. Measurable residual disease detection by high-throughput sequencing improves risk stratification for pediatric B-ALL. Blood. 2018;131(12):1350-9.

16. Huang AJ, Huang CM, Tang GS, et al. Impact of clinical utility of MRD assessment with different techniques on survival in acute B lymphoblastic leukemia. Leukemia Lymphoma. 2018;59(5):1073-83.

17. Coustan-Smith E, Gajjar A, Hijiya N, et al. Clinical significance of minimal residual disease in childhood acute lymphoblastic leukemia after first relapse. Leukemia. 2004;18(3):499-504.

18. Brentjens RJ, Davila ML, Riviere I, et al. CD19-Targeted T Cells Rapidly Induce Molecular Remissions in Adults with Chemotherapy-Refractory Acute Lymphoblastic Leukemia. Sci Transl Med. 2013;5(177).

19. Davila ML, Riviere I, Wang X, et al. Efficacy and Toxicity Management of 19$28 z$ CAR T Cell Therapy in B Cell Acute Lymphoblastic Leukemia. Sci Transl Med. 2014;6(224).

20. Maude SL, Frey N, Shaw PA, et al. Chimeric antigen receptor T cells for sustained remissions in leukemia. N Engl J Med. 2014;371(16):1507-17.

21. Lee DW, Kochenderfer JN, Stetler-Stevenson M, et al. T cells expressing CD19 chimeric antigen receptors for acute lymphoblastic leukaemia in children and young adults: a phase 1 dose-escalation trial. Lancet. 2015; 385(9967):517-28

22. Wen S, Niu Z, Xing L, et al. CAR-T bridging to allo-HSCT as a treatment strategy for relapsed adult acute B-lymphoblastic leukemia: a case report. BMC Cancer. 2018;18(1):1143.

23. Summers C, Annesley C, Bleakley M, Dahlberg A, Jensen MC, Gardner R. Long Term Follow-up after SCRI-CAR19v1 Reveals Late Recurrences As Well As a Survival Advantage to Consolidation with HCT after CAR T Cell Induced Remission. Blood. 2018;132(Suppl 1):967.

24. Taraseviciute A, Broglie L, Phelan R, Bhatt NS, Becktell K, Burke MJ. What is the role of hematopoietic cell transplantation (HCT) for pediatric acute lymphoblastic leukemia (ALL) in the age of chimeric antigen receptor T-cell (CART) therapy? J Pediatr Hematol Oncol. 2019;41(5):337-44.

25. Khazal S, Kebriaei P. Debate: Transplant Is Still Necessary in the Era of Targeted Cellular Therapy for Acute Lymphoblastic Leukemia. Clinical lymphoma, myeloma \& leukemia. 2020 Jun 27 (Epub 2020 Jun 2020.

26. Pan J, Yang JF, Deng BP, et al. High efficacy and safety of low-dose CD19directed CAR-T cell therapy in 51 refractory or relapsed B acute lymphoblastic leukemia patients. Leukemia. 2017;31(12):2587-93.

27. Hay KA, Gauthier J, Hirayama AV, et al. Factors associated with durable EFS in adult B-cell ALL patients achieving MRD-negative CR after CD19 CAR Tcell therapy. Blood. 2019;133(15):1652-63.

28. Jiang $\mathrm{H}$, Li C, Yin P, et al. Anti-CD19 chimeric antigen receptor-modified Tcell therapy bridging to allogeneic hematopoietic stem cell transplantation for relapsed/refractory B-cell acute lymphoblastic leukemia: an open-label pragmatic clinical trial. Am J Hematol. 2019.

29. Jacoby E, Bielorai B, Avigdor A, et al. Locally produced CD19 CAR T cells leading to clinical remissions in medullary and extramedullary relapsed acute lymphoblastic leukemia. Am J Hematol. 2018;93(12):1485-92.

30. Zhang X, Lu XA, Yang JF, et al. Efficacy and safety of anti-CD19 CAR T-cell therapy in 110 patients with B-cell acute lymphoblastic leukemia with highrisk features. Blood Adv. 2020;4(10):2325-38.

31. Lee DW III, Stetler-Stevenson M, Yuan CM, et al. Long-Term Outcomes Following CD19 CAR T Cell Therapy for B-ALL Are Superior in Patients Receiving a Fludarabine/Cyclophosphamide Preparative Regimen and PostCAR Hematopoietic Stem Cell Transplantation. Blood. 2016;128(22):218.

32. Finney OC, Brakke H, Rawlings-Rhea S, et al. CD19 CAR T cell product and disease attributes predict leukemia remission durability. I Clin Investig. 2019; 129(5):2123-32. 
33. Shadman M, Gauthier J, Hay KA, et al. Safety of allogeneic hematopoietic cell transplant in adults after CD19-targeted CAR T-cell therapy. Blood Adv. 2019;3(20):3062-9.

34. Chillon MC, Gomez-Casares MT, Lopez-Jorge CE, et al. Prognostic significance of FLT3 mutational status and expression levels in MLL-AF4+and MLL-germline acute Iymphoblastic leukemia. Leukemia. 2012;26(11):2360-6.

35. Gleissner B, Gokbuget N, Bartram CR, et al. Leading prognostic relevance of the BCR-ABL translocation in adult acute B-lineage lymphoblastic leukemia: a prospective study of the German multicenter trial group and confirmed polymerase chain reaction analysis. Blood. 2002;99(5):1536-43.

36. Vey $\mathrm{N}$, Thomas $\mathrm{X}$, Picard $\mathrm{C}$, et al. Allogeneic stem cell transplantation improves the outcome of adults with $\mathrm{t}(1 ; 19) / \mathrm{E} 2 \mathrm{~A}-\mathrm{PBX} 1$ and $\mathrm{t}(4 ; 11) / \mathrm{MLL}-$ AF4 positive B-cell acute lymphoblastic leukemia: results of the prospective multicenter LALA-94 study. Leukemia. 2006;20(12):2155-61.

37. Forero-Castro M, Robledo C, Benito R, et al. Mutations in TP53 and JAK2 are independent prognostic biomarkers in B-cell precursor acute lymphoblastic leukaemia. Br J Cancer. 2017:117(2):256-65.

38. Rayes A, McMasters RL, O'Brien MM. Lineage switch in MLL-rearranged infant leukemia following CD19-directed therapy. Pediatr Blood Cancer. 2016;63(6):1113-5.

39. Gardner R, Wu D, Cherian S, et al. Acquisition of a CD19-negative myeloid phenotype allows immune escape of MLL-rearranged B-ALL from CD19 CAR-T-cell therapy. Blood. 2016;127(20):2406-10.

40. Oberley MJ, Gaynon PS, Bhojwani D, et al. Myeloid lineage switch following chimeric antigen receptor T-cell therapy in a patient with TCF3-ZNF384 fusion-positive B-lymphoblastic leukemia. Pediatr Blood Cancer. 2018;65(9):5.

41. Nagel I, Bartels M, Duell J, et al. Hematopoietic stem cell involvement in BCR-ABL1-positive ALL as a potential mechanism of resistance to blinatumomab therapy. Blood. 2017;130(18):2027-31.

42. Zhang J, Yang F, Qiu HY, et al. Anti-CD19 chimeric antigen receptors T cells for the treatment of relapsed or refractory E2A-PBX1 positive acute lymphoblastic leukemia: report of three cases. Leukemia Lymphoma. 2019; 60(6):1454-61

43. Kebriaei $P$, Singh $H$, Huls $M H$, et al. Phase I trials using sleeping beauty to generate CD19-specific CAR T cells. J Clin Investig. 2016;126(9):3363-76.

44. Kalos M, Levine BL, Porter DL, et al. T Cells with Chimeric Antigen Receptors Have Potent Antitumor Effects and Can Establish Memory in Patients with Advanced Leukemia. Sci Transl Med. Aug 2011;3(95).

45. Ding J, Karp JE, Emadi A. Elevated lactate dehydrogenase ( $L D H)$ can be a marker of immune suppression in cancer: Interplay between hematologic and solid neoplastic clones and their microenvironments. Cancer Biomarkers. 2017;19(4):353-63.

46. Serganova I, Cohen IJ, Vemuri K, et al. LDH-A regulates the tumor microenvironment via HIF-signaling and modulates the immune response. Plos One. 2018;13(9)

47. Keane C, Tobin J, Talaulikar D, et al. A high LDH to absolute lymphocyte count ratio in patients with DLBCL predicts for a poor intratumoral immune response and inferior survival. Oncotarget. 2018;9(34):23620-7.

48. Kornberg A, Polliack A. Serum Lactic-Dehydrogenase (Ldh) Levels in AcuteLeukemia - Marked Elevations in Lymphoblastic-Leukemia. Blood. 1980;56(3):351-5.

49. Pan L, Beverley PCL, Isaacson PG. Lactate-dehydrogenase (LDH) ISOENZYMES and proliferative activity of lymphoid-cells - an IMMUNOCYTOCHEMICAL study. Clin Exp Immunol. 1991:86(2):240-5.

50. Pui CH, Dodge RK, Dahl GV, et al. Serum Lactic-Dehydrogenase Level Has Prognostic Value In Childhood Acute Lymphoblastic-Leukemia. Blood. 1985; 66(4):778-82.

51. Turtle CJ, Hanafi LA, Berger C, et al. Immunotherapy of non-Hodgkin's lymphoma with a defined ratio of CD8(+) and CD4(+) CD19-specific chimeric antigen receptor-modified T cells. Sci Transl Med. 2016;8(355):12.

52. Ninomiya S, Narala N, Huye L, et al. Tumor indoleamine 2,3-dioxygenase (IDO) inhibits CD19-CAR T cells and is downregulated by lymphodepleting drugs. Blood. 2015;125(25):3905-16.

53. Zhao HL, Wei JP, Wei GQ, et al. Pre-transplant MRD negativity predicts favorable outcomes of CAR-T therapy followed by haploidentical HSCT for relapsed/refractory acute lymphoblastic leukemia: a multi-center retrospective study. J Hematol Oncol. 2020;13(1):13.

54. Jiang H, Liu L, Guo T, et al. Improving the safety of CAR-T cell therapy by controlling CRS-related coagulopathy. Ann Hematol. Jul 2019;98(7):1721-32.

55. Hay KA, Hanafi LA, Li D, et al. Kinetics and biomarkers of severe cytokine release syndrome after CD19 chimeric antigen receptor-modified T-cell therapy. Blood. 2017;130(21):2295-306.
56. Gust J, Hay KA, Hanafi LA, et al. Endothelial activation and blood-brain barrier disruption in neurotoxicity after adoptive immunotherapy with CD19 CAR-T cells. Cancer Discov. 2017;7(12):1404-19.

57. Fabrizio VA, Kernan NA, Boulad F, et al. Low toxicity and favorable overall survival in relapsed/refractory B-ALL following CAR T cells and CD34selected T-cell depleted allogeneic hematopoietic cell transplant. Bone Marrow Transplant. 2020;10.

58. Plasschaert SLA, Kamps WA, Vellenga E, de Vries EGE, de Bont E. Prognosis in childhood and adult acute lymphoblastic leukaemia: a question of maturation? Cancer Treat Rev. 2004;30(1):37-51.

59. Magnani CF, Gaipa G, Lussana F, et al. Sleeping Beauty-engineered CAR T cells achieve anti-leukemic activity without severe toxicities. The Journal of clinical investigation. 2020 Aug 11 (Epub 2020 Aug 2020.

60. Long $A H$, Haso WM, Shern JF, et al. 4-1BB costimulation ameliorates T cell exhaustion induced by tonic signaling of chimeric antigen receptors. Nat Med. 2015;21(6):581-90.

61. Zhao ZG, Condomines M, van der Stegen SJC, et al. Structural Design of Engineered Costimulation Determines Tumor Rejection Kinetics and Persistence of CAR T cells. Cancer Cell. 2015;28(4):415-28.

62. Porter DL, Hwang W-T, Frey NV, et al. Chimeric antigen receptor T cells persist and induce sustained remissions in relapsed refractory chronic lymphocytic leukemia. Sci Transl Med. 2015;7(303).

63. Wang J, Mou N, Yang Z, et al. Efficacy and safety of humanized anti-CD19 CAR-T therapy following intensive lymphodepleting chemotherapy for refractory/relapsed B acute lymphoblastic leukaemia. British journal of haematology. 2020 Mar 31 (Epub 2020 Mar 2020.

64. Pan J, Zuo SY, Deng BP, et al. Sequential CD19-22 CAR T therapy induces sustained remission in children with r/r B-ALL. Blood. Jan 2020;135(5):387-91.

65. Rafiq S, Yeku OO, Jackson HJ, et al. Targeted delivery of a PD-1-blocking scFv by CAR-T cells enhances anti-tumor efficacy in vivo. Nat Biotechnol. 2018;36(9):847.

66. Rupp LJ, Schumann K, Roybal KT, et al. CRISPR/Cas9-mediated PD-1 disruption enhances anti-tumor efficacy of human chimeric antigen receptor T cells. Sci Rep. 2017;7:10.

67. Cherkassky L, Morello A, Villena-Vargas J, et al. Human CAR T cells with cellintrinsic PD-1 checkpoint blockade resist tumor-mediated inhibition. J Clin Investig. 2016;126(8):3130-44.

68. Zhang R, Deng Q, Jiang YY, Zhu HB, Wang J, Zhao MF. Effect and changes in PD-1 expression of CD19 CAR-T cells from T cells highly expressing PD-1 combined with reduced-dose PD-1 inhibitor. Oncol Rep. 2019;41(6):3455-63.

69. Qasim W, Zhan H, Samarasinghe S, et al. Molecular remission of infant BALL after infusion of universal TALEN gene-edited CAR T cells. Sci Transl Med. 2017;9(374):8.

70. Barrett DM, Improving CAR. T cell immunotherapy-mediated remissions for pediatric leukemia. J Clin Investig. 2019;129(5):1842-4.

71. Jacoby E. The role of allogeneic HSCT after CAR T cells for acute lymphoblastic leukemia. Bone Marrow Transplant. 2019;54:810-4.

72. Pan J, Niu Q, Deng B, et al. CD22 CAR T-cell therapy in refractory or relapsed B acute lymphoblastic leukemia. Leukemia. 2019 Dec (Epub 2019 May 2019;33(12):2854-2866.

73. Li L, Liu J, Xu M, et al. Treatment response, survival, safety, and predictive factors to chimeric antigen receptor T cell therapy in Chinese relapsed or refractory B cell acute lymphoblast leukemia patients. Cell Death Dis. 2020; 11(3):207.

74. Zhang $\mathrm{Y}$, Chen $\mathrm{H}$, Song $\mathrm{Y}$, et al. Post-chimeric antigen receptor T-cell therapy haematopoietic stem cell transplantation for 52 cases with refractory/relapsed B-cell acute lymphoblastic leukaemia. Br J Haematol. 2019

\section{Publisher's Note}

Springer Nature remains neutral with regard to jurisdictional claims in published maps and institutional affiliations. 